\title{
Papers
}

\section{Local warming and insertion of peripheral venous cannulas: single blinded prospective randomised controlled trial and single blinded randomised crossover trial}

\author{
Rainer Lenhardt, Tanja Seybold, Oliver Kimberger, Brigitte Stoiser, Daniel I Sessler
}

\begin{abstract}
Objective To determine whether local warming of the lower arm and hand facilitates peripheral venous cannulation.

Design Single blinded prospective randomised controlled trial and single blinded randomised crossover trial.

Setting Neurosurgical unit and haematology ward of university hospital.

Participants 100 neurosurgical patients and 40 patients with leukaemia who required chemotherapy. Interventions Neurosurgical patients' hands and forearms were covered for 15 minutes with a carbon fibre heating mitt. Patients were assigned randomly to active warming at $52^{\circ} \mathrm{C}$ or passive insulation (heater not activated). The same warming system was used for 10 minutes in patients with leukaemia. They were assigned randomly to active warming or passive insulation on day 1 and given alternative treatment during the subsequent visit.

Main outcome measures Primary: success rate for insertion of 18 gauge cannula into vein on back of hand. Secondary: time required for successful cannulation.

Results In neurosurgical patients, it took 36 seconds (95\% confidence interval 31 to 40 seconds) to insert a cannula in the active warming group and 62 (50 to $74)$ seconds in the passive insulation group $(\mathrm{P}=0.002)$. Three $(6 \%)$ first attempts failed in the active warming group compared with $14(28 \%)$ in the passive insulation group $(\mathrm{P}=0.008)$. The crossover study in patients with leukaemia showed that insertion time was reduced by 20 seconds ( 8 to $32, \mathrm{P}=0.013$ ) with active warming and that failure rates at first attempt were $6 \%$ with warming and $30 \%$ with passive insulation $(\mathrm{P}<0.001)$.

Conclusions Local warming facilitates the insertion of peripheral venous cannulas, reducing both time and number of attempts required. This may decrease the time staff spend inserting cannulas, reduce supply costs, and improve patient satisfaction.
\end{abstract}

\section{Introduction}

Insertion of intravenous cannulas is probably the most commonly performed invasive medical procedure.
Insertion is usually technically easy and causes patients only mild distress, but sometimes it is problematic and time consuming. ${ }^{1}$ Cannula insertion is notoriously difficult in intravenous drug users and patients having repeated courses of chemotherapy. The procedure is also difficult in infants and children, obese patients, and black people. It is often complicated in patients who are afraid of needles or have had bad experiences because fear activates the sympathetic nervous system thereby provoking peripheral vasoconstriction. ${ }^{2}$ Once an initial attempt has failed, nearly all patients experience a degree of sympathetic activation that makes subsequent attempts increasingly difficult. Failed attempts are also embarrassing for the provider. It is therefore important that a cannula is inserted quickly on the first attempt.

To facilitate insertion the hand and lower arm can be warmed with various techniques such as wrapping in towels moistened with hot water ${ }^{3}$ or immersing in hot water. We are not aware of any prospective randomised trials on warming the skin to facilitate venous cannula insertion.

Carbon fibre resistive heating has been used for warming patients before admission to hospital and before and after operations. ${ }^{4}$ We developed a special "warming mitt" designed to warm the hand and lower arm (figure). We examined whether such warming speeds venous cannulation and improves success rates.

\section{Methods}

Our study was approved by the University of Vienna's institutional review board. We obtained written informed consent from all participants. Patients were informed that we were testing a device to facilitate insertion of an intravenous cannula, though they were not told that warming was the key factor. All procedures were performed at a general hospital. Ambient temperature was maintained near $23^{\circ} \mathrm{C}$.

We initially studied 100 neurosurgical patients because a preliminary study indicated that this number would provide an $80 \%$ chance of identifying a $15 \%$ reduction in failure at the first attempt in the warmed group with a two tailed $\alpha$ of 0.05 . Patients who had a physical status score of 1 or 2 (healthy or mild and well controlled systemic disease, American Society of Anesthesiologists) were eligible to participate.

Outcomes Research
Institute and
Department of
Anaesthesiology,
University of
Louisville,
Louisville, KY
40202 USA
Rainer Lenhardt
assistant professor
Daniel I Sessler
associate dean for
research
Department of
Anaesthesiology,
University of
Vienna, Vienna
A-1090, Austria
Tanja Seybold
resident in
anaesthesia
Oliver Kimberger
resident in
anaesthesia
Department of
Internal Medicine,
University of
Vienna
Brigitte Stoiser
resident in
haematology
Correspondence to:
R Lenhardt
rainerlenhardt@
compuserve.com
bmj.com 2002;325:409

bmj.com 2002;325:409 
On the basis of the data from neurosurgical patients we calculated that we would need 40 patients in a crossover trial to provide an $80 \%$ chance of identifying a significant difference between the groups with a two tailed $\alpha$ of 0.05 . We carried out this part of the study in patients with leukaemia who were scheduled for at least two sessions of chemotherapy, at least one week apart. In these patients the mean weight was 71 (SD 19) $\mathrm{kg}$, the mean height was 169 (SD 8) $\mathrm{cm}$, and the mean age was 63 (SD 14) years; 20 were men.

\section{Protocol}

A carbon fibre warming mitt (Thermamed, Bad Oeynhausen, Germany), consisting of a carbon fibre resistive heating element covered with cloth, was placed over the left hand and forearm of the neurosurgical patients in the preoperative area. The mitt is connected by a wire to a solid state controller. It has been approved by the German Technische Überprüfungsanstalt TÜF (technical monitoring agency) and has CE certification (safety certificate from the European Union), confirmed by Thermamed. However, it has not been approved by the US Food and Drug Administration and is not commercially available in the rest of Europe. There is no shock hazard associated with this device as it is powered by 12 volts DC. The mitt is closed on three sides, leaving the fourth side open for insertion of the cannula.

Participating neurosurgical patients were randomly assigned to passive warming (mitt not heated) or to active warming (mitt warmed to $52^{\circ} \mathrm{C}$ ). Group assignment was determined by computer generated codes, thus ensuring an equal distribution of participants. Codes were kept in sequentially numbered opaque envelopes until just before use. The mitt was removed after 15 minutes and patients were asked to clench their left hands. A tourniquet was then applied $10 \mathrm{~cm}$ proximal to the wrist and the patient relaxed his or her hand. A nurse anaesthetist, blinded to treatment group, then attempted to insert an 18 gauge cannula into a vein on the back of the left hand. The nurse anaesthetist was not otherwise involved in the study and was not told that local warming was the basis for our hypothesis. The same nurse anaesthetist attempted all cannulations.

In the patients with leukaemia the warming mitt was positioned over the hand and lower arm for only 10 minutes as the physician in charge of the ward would not permit a longer delay. This allowed us to

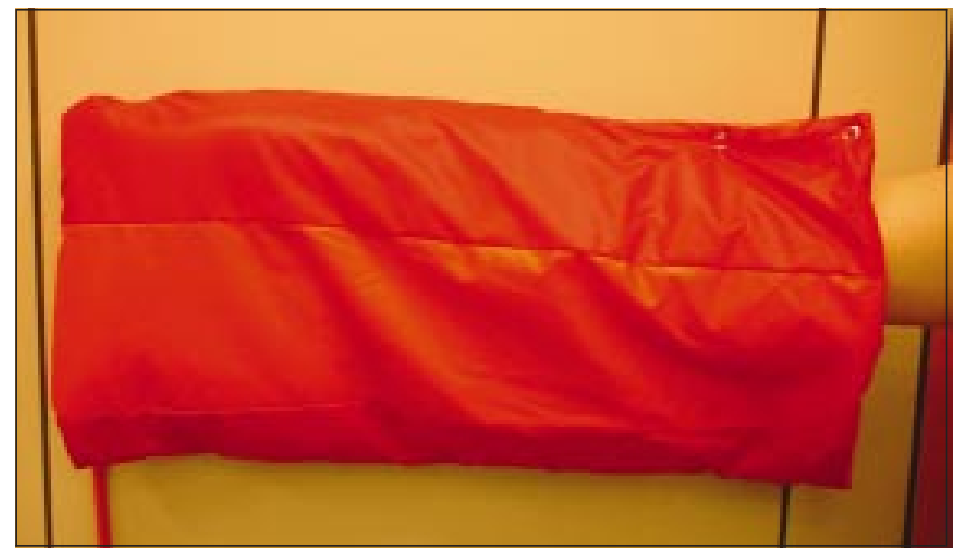

Warming mitt in use determine if a shorter and therefore more practical period would be adequate. Patients were initially randomly assigned to either passive insulation (mitt not heated) or to active warming (mitt warmed to $52^{\circ} \mathrm{C}$ ); the alternative treatment was then applied at their next chemotherapy visit. After the mitts were removed patients were asked to clench their left hands and in each a tourniquet was applied $10 \mathrm{~cm}$ proximal to the wrist and the hand relaxed. A resident in haematology, blinded to treatment, then attempted to insert an 18 gauge cannula. The residents were not otherwise involved in the study and were not told that local warming was the basis for our hypothesis.

In both groups of patients we ended the study after the first successful attempt at cannulation. Subsequent management was at the discretion of the provider.

\section{Measurements}

We asked patients about their thermal comfort before the warming mitt was put on and before it was taken off. We scored thermal comfort using a $100 \mathrm{~mm}$ visual analogue scale with $0 \mathrm{~mm}$ indicating the worst imaginable cold, $50 \mathrm{~mm}$ identifying thermoneutrality, and $100 \mathrm{~mm}$ being the worst imaginable heat.

Vein status was evaluated as veins neither visible nor palpable, veins visible but not palpable, veins barely visible and palpable, veins visible and palpable, and veins clearly visible and easily palpable. Assessments were made by the nurse anaesthetist or haematology resident, both of whom were blinded to treatment. Veins were evaluated before and after the treatment period but before the first attempt at cannulation.

We recorded skin temperatures on the forearm, the back of the hand, and the fingertip of the treated arm with Mon-a-Therm thermocouples (Mallinckrodt Anesthesiology Products, St Louis, MO) at the beginning and end of the warming period in both groups.

We measured the time from the start of searching for an appropriate vein (after the tourniquet was applied) to successful insertion of the cannula. Successful cannulation was confirmed by administration of a crystalloid solution without any signs of infiltration. Likewise, we recorded the number of failed first attempts. We examined the treated hand and arm for burns or skin irritation after the cannula had been inserted. We asked the neurosurgical patients if they had experienced any thermal discomfort related to the treatment.

\section{Analysis}

We analysed the results after all the data were collected. An audit confirmed integrity of the randomisation process and that the computer generated codes were used in sequential order. We compared data from the warmed and unwarmed neurosurgical patients with unpaired two tailed $t$ or $\chi^{2}$ tests. We compared results in the warmed and unwarmed patients with leukaemia with paired two tailed $t$ or McNemar tests. We have presented data as means (SD); $\mathrm{P}<0.05$ identified significant differences.

\section{Results}

\section{Neurosurgical patients}

All enrolled patients completed the study. Characteristics of patients were comparable in the actively and passively warmed groups. Before warming there was a 
significant but clinically unimportant difference in hand temperatures. However, forearm and fingertip temperatures and vein scoring were comparable.

Fifteen minutes of warming significantly increased vein scores, thermal comfort, and skin temperatures (table 1). The success rate for insertion of the intravenous cannula was $94 \%(44 / 50)$ in the active warming group versus $72 \%(36 / 50)$ in the passive insulation group $(\mathrm{P}=0.008)$. Cannula insertion took about half as long with active warming (36 seconds (95\% confidence interval 31 to 40 seconds) versus 62 seconds (50 to 74 seconds), table 1 ). We did not observe any skin irritation nor did any patients report any discomfort

\section{Patients with leukaemia}

We enrolled 42 patients with leukaemia. Two failed to return for their subsequent course of chemotherapy or declined to participate further in the study; both had initially been assigned to passive insulation. Thus 40 patients completed both study days. Only these patients were included in the data analysis.

Ten minutes of active warming significantly increased vein scores, thermal comfort, and skin temperatures (table 2). On the day patients were assigned to active warming, the success rate for insertion of the cannula in these patients was 95\% versus $73 \%$ in the passive warming group $(\mathrm{P}<0.001)$. The time elapsing from beginning to search for an appropriate vein until successful cannulation was 20 seconds (8 to 32) shorter with active warming than with passive insulation ( $\mathrm{P}=0.02$, table 2$)$. We did not observe any skin irritation.

\section{Discussion}

Percutaneous intravenous cannulation is usually rapid, but because the procedure is so common even modest reductions in the time required could be clinically important. Various procedures improve venous visibility and thus, presumably, facilitate insertion of intravenous cannulas. For example, vein prominence is usually increased by gently tapping over the site, applying proximal tourniquets, or asking patients to clench and relax their hands. ${ }^{67}$ Venous engorgement may be improved by having arm hanging down. These measures are used routinely but often fail to augment vein size sufficiently.

In our patients local warming increased vein size. This increase was clinically important in that it halved insertion time in both groups. Equally importantly, the fraction of unsuccessful attempts was reduced fivefold. Warming with topical application of $4 \%$ nitroglycerine ointment also decreases the number of cannulation attempts ${ }^{8}$ and facilitates insertion. ${ }^{9}$ However, topical nitroglycerine is systemically active and requires considerably longer to produce venodilation than local heating.

Local heating increased hand temperature and produced a local warm sensation. However, skin temperature averaged only about $40.5^{\circ} \mathrm{C}$, even after 15 minutes of warming. This temperature is not associated with tissue injury as long as it is not combined with prolonged pressure. ${ }^{10} 11$ None of the volunteers reported discomfort, and none experienced cutaneous irritation. We therefore conclude that local
Table 1 Details of neurosurgical patients in trial of effect of warming on cannulation. Figures are mean (SD) unless stated otherwise

\begin{tabular}{|c|c|c|c|}
\hline & $\begin{array}{l}\text { Active warming } \\
\qquad(\mathrm{n}=50)\end{array}$ & $\begin{array}{l}\text { Passive insulation } \\
\qquad(\mathrm{n}=50)\end{array}$ & $P$ value \\
\hline Men & 21 & 26 & $0.42^{*}$ \\
\hline Age (years) & $56(15)$ & $50(14)$ & $0.06^{*}$ \\
\hline Weight (kg) & $74(13)$ & $72(13)$ & $0.32^{*}$ \\
\hline Height (cm) & $170(8)$ & $170(9)$ & $0.72^{*}$ \\
\hline \multicolumn{4}{|l|}{ Vein score $†>3$ before treatment: } \\
\hline No $(\%)$ of patients & $12(24)$ & $12(24)$ & \\
\hline Thermal comfort before treatment (VAS) & $51(5)$ & $46(6)$ & \\
\hline Fingertip temperature before treatment $\left({ }^{\circ} \mathrm{C}\right)$ & $31.7(3.1)$ & $30.9(3.1)$ & \\
\hline Hand temperature before treatment $\left({ }^{\circ} \mathrm{C}\right)$ & $32.7(2.5)$ & $31.6(1.9)$ & \\
\hline Forearm temperature before treatment $\left({ }^{\circ} \mathrm{C}\right)$ & $33(1.8)$ & $32.7(1.1)$ & \\
\hline \multicolumn{4}{|l|}{ Vein score $\dagger>3$ after treatment: } \\
\hline No $(\%)$ of patients & $35(30)$ & $15(70)$ & $<0.0001 \ddagger$ \\
\hline Thermal comfort after treatment (VAS) & $58(8)$ & $46(10)$ & $<0.001^{*}$ \\
\hline Fingertip temperature after treatment $\left({ }^{\circ} \mathrm{C}\right)$ & $40.5(2.1)$ & $33.0(3.6)$ & $<0.001^{*}$ \\
\hline Hand temperature after treatment $\left({ }^{\circ} \mathrm{C}\right)$ & $40.6(2.3)$ & $33.3(1.6)$ & $<0.001^{*}$ \\
\hline Forearm temperature after treatment $\left({ }^{\circ} \mathrm{C}\right)$ & $38.0(1.9)$ & $34.5(0.9)$ & $0.03^{*}$ \\
\hline No (\%) of failed first attempts & $3(6)$ & $14(28)$ & $0.008 \ddagger$ \\
\hline Mean $(95 \% \mathrm{Cl})$ time to successful insertion (s) & 36 (31 to 40$)$ & $62(50$ to 74$)$ & $0.002^{*}$ \\
\hline
\end{tabular}

VAS=visual analogue score

${ }^{*} t$ test.

†Scores: $1=$ veins not visible or palpable, $2=$ veins visible but not palpable, $3=$ veins barely visible and palpable, $4=$ veins visible and palpable, $5=$ veins clearly visible and easily palpable. $\ddagger \chi^{2}$ test.

Table 2 Differences between cannulation with active warming and passive insulation in patients with leukaemia in crossover trial. Figures are means (95\% confidence intervals) or number of patients

\begin{tabular}{|c|c|c|}
\hline & $\begin{array}{l}\text { Differences within } \\
\text { patients }(n=40)\end{array}$ & $P$ value \\
\hline $\begin{array}{l}\text { Patients with vein score }{ }^{\star}>3 \text { with active warming but not with } \\
\text { passive insulation }\end{array}$ & 12 & $0.0027 \dagger$ \\
\hline $\begin{array}{l}\text { Patients with vein score }{ }^{*}>3 \text { with passive insulation but not with } \\
\text { active warming }\end{array}$ & 0 & $0.0027 \dagger$ \\
\hline Patients with same vein score ${ }^{*}$ grouping regardless of treatment & 28 & $0.0027 \dagger$ \\
\hline Difference in fingertip temperature $\left({ }^{\circ} \mathrm{C}\right)$ & 4.7 (3.7 to 5.8$)$ & $<0.0001 \ddagger$ \\
\hline Difference in hand temperature $\left({ }^{\circ} \mathrm{C}\right)$ & $5.3(4.5$ to 6.0$)$ & $<0.0001 \ddagger$ \\
\hline Difference in forearm temperature $\left({ }^{\circ} \mathrm{C}\right)$ & $3.3(2.5$ to 4.0$)$ & $<0.0001 \ddagger$ \\
\hline $\begin{array}{l}\text { Patients with failed first attempts for passive insulation but success } \\
\text { with active warming }\end{array}$ & 10 & $0.0067 \dagger$ \\
\hline $\begin{array}{l}\text { Patients with failed first attempts for active warming but success } \\
\text { with passive insulation }\end{array}$ & 1 & $0.0067 \dagger$ \\
\hline Patients with same results regardless of treatment & 29 & $0.0067 \dagger$ \\
\hline Difference in time to successful insertion (s) & 20 (8 to 32$)$ & $0.0019 \ddagger$ \\
\hline
\end{tabular}

* See table 1 for definition of vein scoring.

†McNemar test.

†Paired $t$ test.

warming is safe as well as effective. In the patients with leukaemia we were allowed to warm patients' hands for only 10 minutes and thus skin temperatures were lower. However, fewer attempts were needed for successful cannula insertion in the warmed group. Although we were testing different groups of patients it seems likely that warming patients' hands and arms for just 10 minutes will increase the rate of successful first attempts.

\section{Possible bias}

Arteriovenous shunt blood flow and hand venous tone is not normally under conscious control. Furthermore, patients were not informed that we were testing warming (as opposed to passive insulation or some other aspect of the device). It thus seems unlikely that our results were biased by responses under the patients' control. 


\section{What is already known on this topic}

Insertion of peripheral venous cannulas may be difficult because of severe vasoconstriction

Vasoconstriction can be overcome by local heating

\section{What this study adds}

Active local warming facilitates the insertion of peripheral venous cannulas, reducing both the time and number of attempts required

Local warming will decrease the amount of time staff spend inserting cannulas, reduce supply costs, and improve patient satisfaction

The nurse anaesthetist and residents who attempted to insert the intravenous cannulas were blinded to treatment and were also not told that we were testing warming. Obviously, they could feel that in some patients the hands were warmed, and to this extent our blinding was unavoidably incomplete. However, we consider that the observed differences in cannula insertion time and success rates result from local warming rather than investigator bias.

Contributors: RL initiated and coordinated the formulation of the primary study hypothesis, designed the protocol, analysed the data, and wrote the paper. TS participated in data collection and documentation of the neurosurgical patients. OK and BS participated in data collection and documentation of the haematological patients. DIS participated in the design of the study protocol and discussed core ideas and interpretation of the findings.

Funding: NIH grant GM58273 (Bethesda, MD), Drown Foundation (Los Angeles, CA), Commonwealth of Kentucky Research Challenge Trust Fund (Louisville, KY), and Anniversary Fund of the Austrian National Bank. Thermamed, GmbH. (Bad Oeynhausen, Germany) donated the warming mitt. Mallinckrodt (St Louis, MO) donated the thermocouples and thermometers.

Competing interests: DIS is a consultant for ThermaMed.

1 Cleary M. Peripheral intravenous cannulation. Aust Fam Physician 1991;20:1285-8.

2 Johnstone M. The effect of lorazepam on the vasoconstriction of fear Anaesthesia 1976;31:868-72.

3 Mbamalu D, Banerjee A. Methods of obtaining peripheral venous access in difficult situations. Postgrad Med J 1999;75:459-62.

4 Greif R, Rajek A, Laciny S, Bastanmehr H, Sessler D. Resistive heating is more effective than metallic-foil insulation in an experimental model of accidental hypothermia: a randomized controlled trial. Ann Emerg Med 2000;35:337-45

5 Kober A, Scheck T, Fulesdi B, Lieba F, Vlach W, Friedmann A, et al. Effectiveness of resistive heating compared with passive warming in treating hypothermia associated with minor trauma: a randomized trial. Mayo Clin Proc 2001;76:369-75.

6 Datta S, Hanning CD. How to insert a peripheral venous cannula. $\mathrm{Br} J$ Hosp Med 1990;43:67-9.

7 Roberts GH, Carson J. Venipuncture tips for radiologic technologists. Radiol Technol 1993;65:107-12; quiz 113-5.

8 Roberge RJ, Kelly M, Evans TC, Hobbs E, Sayre M, Cottington E. Facilitated intravenous access through local application of nitroglycerin ointment. Ann Emerg Med 1987;16:546-9.

9 Michael A, Andrew M. The application of EMLA and glyceryl trinitrate ointment prior to venepuncture. Anaesth Intensive Care 1996;24:360-4.

10 Morris CC, Field SB. The relationship between heating time and temperature for rat tail necrosis with and without occlusion of the blood supply. Int J Radiat Biol 1985;47:41-8.

11 Gendron FG. Unexplained patient burns: investigating iatrogenic injuries. Brea, CA: Quest Publishing, 1988.

(Accepted 2 April 2002) 